\title{
The impact of NHS Health Checks on the prevalence of disease in general practices:
}

\author{
a controlled study
}

\begin{abstract}
Background

NHS Health Checks is a national case-finding and vascular risk assessment programme in England. No research has been published to assess the impact of NHS Health Checks on the prevalence of chronic disease in GP practices.

\section{Aim}

To examine the impact of NHS Health Checks on the prevalence of hypertension, coronary heart disease (CHD), chronic kidney disease (CKD), atrial fibrillation (AF), and diabetes within practices, and compare this with usual medical care.
\end{abstract}

\section{Design and setting}

A non-randomised controlled study in a mixed rural and urban county in England.

\section{Method}

Thirty-eight GP practices provided NHS Health Checks over a 3-year period. Forty-one practices that did not provide Health Checks acted as controls. $t$-tests and multiple linear regression were used to assess the difference in prevalence of disease between intervention group and control group practices, and the impact of NHS Health Checks on this.

\section{Results}

Throughout the duration of the study, 1142 previously undiagnosed cases of disease were detected through a total of 16669 NHS Health Checks. Despite this, there were no significant differences in the change to the prevalence of diabetes, hypertension, CHD, CKD, and $\mathrm{AF}$ in practices providing NHS Health Checks compared with control practices. Regression analysis did not demonstrate that there was any significant association between the proportion of the eligible population of a practice having completed NHS Health Checks and changes in the prevalence of the five conditions studied.

\section{Conclusion}

In practices providing NHS Health Checks, the change in the reported prevalence of diabetes, hypertension, CHD, CKD, and AF did not differ from that of practices providing usual care.

\section{Keywords}

cardiovascular disease, health checks, primary care, risk factors, screening.

\section{INTRODUCTION}

In April 2009 the Department of Health introduced 'NHS Health Checks' in England. This is a national systematic case-finding and vascular risk assessment programme. It is offered to those between the ages of 40 and 74 years without a prior diagnosis of cardiovascular disease (CVD) or other risk factors. The aim of NHS Health Checks is to identify and treat patients at high risk of developing CVD and to identify undiagnosed CVD, diabetes, hypertension, and chronic kidney disease (CKD) to reduce the risk of future illness, and had been modelled to reduce future healthcare demand. ${ }^{1}$ Vascular diseases including coronary heart disease (CHD), diabetes, stroke, and CKD account for the greatest number of preventable deaths in the UK. ${ }^{2}$ In 2009, one-third of deaths and one in five hospital admissions in the UK were attributable to CVD. ${ }^{3}$

NHS Health Checks are usually carried out by staff, often a nurse or a trained healthcare assistant, in a patient's usual GP practice using a nationally specified protocol. Each Health Check should last 20-30 minutes and consist of a personal history including: age, ethnic group, smoking status, family history, assessment of physical activity, measurement of body mass index, blood pressure, smoking status, renal function, lipid levels, and blood glucose where indicated. The patient's risk of developing cardiovascular disease is then calculated using the CVD risk calculator QRISK. ${ }^{4}$ Those with a greater

M Caley, MPH, MRCP, MFPH, DTM\&H, specialist registrar in public health and general practice; P Chohan, MBBS, FY2 doctor; J Hooper, MBBS, FY2 doctor; N Wright, MPH, MFPH, consultant in public health, Public Health Warwickshire, Warwick.

\section{Address for correspondence}

Michael Caley, Public Health Warwickshire,

PO Box 43 - Shire Hall, Barrack Street, Warwick, than 20\% risk of developing CVD over 10 years are deemed high risk and are offered preventive treatment as per NICE guidance. ${ }^{1}$ If a diagnosable condition is detected, the patient is referred to a GP for further assessment and treatment, and is also placed on the practice's appropriate disease register. The numbers of patients on certain specified disease registers for each practice in England are nationally collected and published annually in the Quality and Outcomes Framework (QOF).

Many of the interventions within NHS Health Checks and the early detection and intervention for certain conditions are well supported by evidence to improve outcomes for individual patients. NHS Health Checks as a programme, however, was implemented with little direct evaluation of the benefit of the programme as a vehicle to offer these interventions to the general public. There has been a good deal of debate about the relative worth of NHS Health Checks and, although the Department of Health has commissioned some research to evaluate the impact of the programme, ${ }^{5}$ at present little published evidence exists on the direct outcomes of the NHS Health Checks programme. No research has been published that examines the effect of NHS Health Checks on the prevalence of disease within participating practices.

This study examines changes in prevalence of diabetes, hypertension, coronary heart disease (CHD), chronic kidney disease (CKD), and atrial fibrillation

CV34 4SX, UK

E-mail: mikecaleydadoctors.org.uk

Submitted: 27 December 2013; Editor's response: 5 February 2014; final acceptance: 1 April 2014 (CBritish Journal of General Practice

This is the full-length article (published online 28 July 2014) of an abridged version published in print. Cite this article as: Br J Gen Pract 2014; DOI: 10.3399/bjgp14X681013 


How this fits in
NHS Health Checks is a national case-
finding and vascular risk assessment
programme in England. No previous
research has explored the impact of NHS
Health Checks on the prevalence of the
conditions that the programme aims
to case find, namely diabetes, coronary
heart disease, chronic kidney disease,
hypertension, and atrial fibrillation. This
study was unable to show any difference
in the change in prevalence of these
conditions between practices providing
NHS Health Checks and practices
providing usual medical care.

(AF) in GP practices that have implemented NHS Health Checks compared with control practices, and estimates the effect that NHS Health Checks has had on this change.

\section{METHOD}

Data were used from 38 GP practices in Warwickshire, a mixed rural and urban county in the Midlands, England, which provided NHS Health Checks over a 3-year period between June 2010 and March 2013. Forty-one GP practices within Coventry and Warwickshire did not provide NHS Health Checks during the study period and were used as control practices, providing usual medical care.

Data regarding NHS Health Checks were collected from each practice, including the number of NHS Health Checks offered, the number of NHS Health Checks completed, and the number of new cases of diabetes, hypertension, CHD, CKD, and $A F$ detected as a result of the Health

\section{Table 1. Descriptive statistics of intervention and control practices}

\begin{tabular}{|c|c|c|c|}
\hline & $\begin{array}{l}\text { Intervention } \\
\text { practices }\end{array}$ & $\begin{array}{l}\text { Control } \\
\text { practices }\end{array}$ & $P$-value \\
\hline Number of practices & 38 & 41 & \\
\hline Mean list size (range) & 7207 (1751-19 603) & 7378 (189 816-247) & 0.990 \\
\hline $\begin{array}{l}\text { Mean number of patients eligible } \\
\text { for health check (range) }\end{array}$ & $3226(753-7617)$ & $3356(894-7840)$ & 0.760 \\
\hline Mean age, years (range) & $40.76(29.28-46.16)$ & $41.50(30.42-46.45)$ & 0.310 \\
\hline Males, \% (range) & $0.50(0.48-0.57)$ & $0.50(0.46-0.58)$ & 0.480 \\
\hline Mean deprivation (IMD) score (range) & $19.89(8.00-38.79)$ & $12.47(5.60-32.83)$ & $<0.001$ \\
\hline Total Health Checks offered & 37236 & 0 & \\
\hline Total Health Checks completed & 16669 & 0 & \\
\hline Eligible offered Health Check, \% & 30.38 & - & \\
\hline Eligible completed Health Check, \% & 13.60 & - & \\
\hline Offered completed Health Check, \% & 44.77 & - & \\
\hline
\end{tabular}

Checks. Information was collected on a quarterly basis throughout the study period by the commissioner of the Health Checks programme. The programme did not specifically determine the diagnostic criteria for the five conditions but relied on practices to determine and report a case of disease using their usual diagnostic criteria. Cases of disease that may have been detected within the intervention practices through a route other than NHS Health Checks, that is usual medical care, were not reported by practices to the commissioner. This was because they did not constitute the intervention under examination, although these cases would be captured in the practices' overall prevalence rates for individual conditions.

The prevalences of CVD, diabetes, hypertension, AF, and CKD were obtained for every practice in the study from the disease registers maintained under the Quality and Outcomes Framework (QOF), which is a national standard dataset. ${ }^{6}$ QOF is a national annual incentive programme for all GP surgeries in England and includes practice-level disease prevalence for several conditions as well as performance against nationally specified criteria. Prevalence rates were obtained for the financial year 2009-2010 (ending March 2010) and represent the baseline prevalence for the study. Prevalence figures for the financial year 2012-2013 (ending March 2013) were obtained and represent the results at the end of the study period.

Descriptive statistics, including practice list size, mean age of practice population, proportion of the practice population that were male, and practice deprivation score were calculated for intervention and control practices, with $t$-tests applied to show the significance of any differences. These tests were also applied to the starting prevalence and final prevalence for the five conditions studied to assess the significance of change in prevalence over the study period.

Multiple linear regression analysis was used to estimate the association between change in the prevalence of five individual conditions over the study period and six variables: practice list size, mean age of practice population, proportion of the practice population that were male, practice deprivation score (Index of Multiple Deprivation 2010), baseline prevalence of disease, and proportion of eligible patients with a completed NHS Health Check. The proportion of the eligible population who had had a completed NHS Health Check was chosen as the most meaningful measure of the volume of NHS Health Checks carried out and, as such, the most 


\section{Table 2. Cases of disease detected by NHS Health Checks in the intervention group}

\begin{tabular}{lccc} 
& $\begin{array}{c}\text { Case detected in } \\
\text { intervention } \\
\text { practices by NHS } \\
\text { Health Checks }\end{array}$ & $\begin{array}{c}\text { Proportion } \\
\text { of all Health } \\
\text { checks, \% }\end{array}$ & $\begin{array}{c}\text { Mean cases } \\
\text { per practice } \\
\text { (range) }\end{array}$ \\
\hline Condition & 210 & 1.26 & $6.00(0-26)$ \\
Diabetes & 635 & 3.81 & $18.14(0-88)$ \\
Hypertension & 198 & 1.19 & $5.66(0-34)$ \\
Chronic kidney disease & 43 & 0.26 & $1.23(0-7)$ \\
Coronary heart disease & 56 & 0.34 & $1.60(0-9)$ \\
Atrial fibrillation & 1142 & 6.85 & \\
Total & & & \\
\hline
\end{tabular}

\section{Table 3. Mean baseline and final prevalence for five conditions and change in prevalence over the study period}

\begin{tabular}{|c|c|c|c|}
\hline & $\begin{array}{l}\text { Intervention } \\
\text { group, } \%\end{array}$ & $\begin{array}{l}\text { Control } \\
\text { group, \% }\end{array}$ & $P$-value \\
\hline \multicolumn{4}{|l|}{ Diabetes } \\
\hline Baseline prevalence (2009-2010) & 5.90 & 4.56 & $<0.001^{a}$ \\
\hline Final prevalence (2012-2013) & 5.50 & 4.19 & $<0.001^{a}$ \\
\hline Change in prevalence & -0.40 & -0.37 & 0.747 \\
\hline \multicolumn{4}{|l|}{ Hypertension } \\
\hline Baseline prevalence (2009-2010) & 15.26 & 14.14 & 0.088 \\
\hline Final prevalence (2012-2013) & 15.71 & 14.44 & 0.053 \\
\hline Change in prevalence & +0.46 & +0.30 & 0.544 \\
\hline \multicolumn{4}{|l|}{ Atrial fibrillation } \\
\hline Baseline prevalence (2009-2010) & 1.30 & 1.57 & $0.002^{\mathrm{a}}$ \\
\hline Final prevalence (2012-2013) & 1.40 & 1.73 & $0.001^{\mathrm{a}}$ \\
\hline Change in prevalence & +0.10 & +0.15 & 0.514 \\
\hline \multicolumn{4}{|l|}{ Chronic kidney disease } \\
\hline Baseline prevalence (2009-2010) & 4.90 & 4.48 & 0.448 \\
\hline Final prevalence (2012-2013) & 4.30 & 4.10 & 0.636 \\
\hline Change in prevalence & -0.60 & -0.38 & 0.542 \\
\hline \multicolumn{4}{|l|}{ Coronary heart disease } \\
\hline Baseline prevalence (2009-2010) & 3.30 & 3.22 & 0.510 \\
\hline Final prevalence (2012-2013) & 3.20 & 3.11 & 0.660 \\
\hline Change in prevalence & -0.10 & -0.10 & 0.639 \\
\hline
\end{tabular}

meaningful explanatory variable.

G*Power (version 3.1.9) was used to calculate the required sample size to detect a difference of a $2 \%$ change in prevalence between the two groups using multiple linear regression with six dependent variables. Assuming a required power of $80 \%$ and at a significance level of $95 \%$, then a sample size of 311 practices would be required. ${ }^{7}$ Data on the number of health checks completed and the number of cases detected were only available for practices within Warwickshire, however which limited the ability to expand the sample size. Seventy-nine practices were included in the study, which resulted in the study having an actual power to detect this difference of $34.5 \%$.

\section{RESULTS}

The intervention and control groups were reasonably well matched with no significant difference between them in terms of their list size, mean number of patients eligible for a health check, the mean age of registered patients, the proportion of registered patients that were male, and practice deprivation scores. Practices in the intervention group had significantly higher deprivation scores (that is, more deprived) than practices in the control group (Table 1).

A total of 1142 new cases of disease were detected through the NHS Health Checks programme (Table 2), equivalent to a case of disease being detected in $6.85 \%$ of all Health Checks.

There were significant differences between the two groups in the baseline and final prevalences for both diabetes and atrial fibrillation (Table 3). No statistically significant difference was found in the percentage point change in prevalence, however, for any of the conditions studied over the study period between the two groups. No obvious trend in superiority was observed for either group.

Table 4 shows the results of five multiple regression analyses that assess the association between the change in prevalence of the five conditions studied and proportion of the eligible population with a completed NHS Health Check, the practice list size, the baseline prevalence of the condition, the mean age of registered patients, the proportion of registered patients that were male, and practice deprivation scores. Baseline prevalence of disease was consistently and strongly negatively correlated with an increasing change in prevalence in all five conditions over the study period. For all five conditions increasing mean age of the practice population had a consistently significant but weak positive correlation with increasing change in prevalence. Significant but comparatively weak correlations were observed between list size, deprivation, and the proportion of the patients who were male and change in the prevalence of diabetes, and between deprivation and the proportion of the patients who were male and change in the prevalence of hypertension. There was no significant correlation observed 


\section{Table 4. Results of multiple regression models examining the association between the change in prevalence of the five conditions between 2009-2010 and 2012-2013 and the six variables}

\begin{tabular}{|c|c|c|c|}
\hline & Coefficients & $95 \% \mathrm{Cl}$ & $P$-value \\
\hline \multicolumn{4}{|l|}{ Diabetes } \\
\hline $\mathrm{R}^{2}$ & 0.427 & & \\
\hline Adjusted $\mathrm{R}^{2}$ & 0.379 & & \\
\hline Standard error & 0.004 & & \\
\hline Intercept & -0.107 & -0.140 to -0.074 & $<0.001^{\mathrm{a}}$ \\
\hline List size & 0.000 & 0.000 to 0.000 & $0.042^{a}$ \\
\hline Mean age & 0.001 & 0.000 to 0.001 & $0.044^{\mathrm{a}}$ \\
\hline$\%$ Male & 0.133 & 0.076 to 0.189 & $<0.001^{\mathrm{a}}$ \\
\hline \% Eligible completing a Health Check & 0.000 & 0.000 to 0.001 & 0.057 \\
\hline Deprivation score (IMD) & 0.000 & 0.000 to 0.001 & $0.001^{a}$ \\
\hline Baseline prevalence (2009-2010) & -0.198 & -0.295 to -0.101 & $<0.001^{a}$ \\
\hline \multicolumn{4}{|l|}{ Hypertension } \\
\hline $\mathrm{R}^{2}$ & 0.372 & & \\
\hline Adjusted $\mathrm{R}^{2}$ & 0.320 & & \\
\hline Standard error & 0.009 & & \\
\hline Intercept & -0.159 & -0.238 to -0.079 & $<0.001^{a}$ \\
\hline List size & 0.000 & 0.000 to 0.000 & 0.139 \\
\hline Mean age & 0.002 & 0.001 to 0.004 & $0.005^{a}$ \\
\hline$\%$ Male & 0.151 & 0.022 to 0.280 & $0.023^{a}$ \\
\hline$\%$ Eligible completing a Health Check & 0.001 & 0.000 to 0.002 & 0.131 \\
\hline Deprivation score (IMD) & 0.001 & 0.000 to 0.001 & $0.002^{\mathrm{a}}$ \\
\hline Baseline prevalence (2009-2010) & -0.264 & -0.362 to -0.166 & $<0.001^{a}$ \\
\hline \multicolumn{4}{|l|}{ Atrial fibrillation } \\
\hline $\mathrm{R}^{2}$ & 0.199 & & \\
\hline Adjusted $\mathrm{R}^{2}$ & 0.132 & & \\
\hline Standard error & 0.002 & & \\
\hline Intercept & -0.005 & -0.019 to 0.009 & 0.499 \\
\hline List size & 0.000 & 0.000 to 0.000 & 0.726 \\
\hline Mean age & 0.000 & 0.000 to 0.001 & $0.015^{a}$ \\
\hline$\%$ Male & -0.008 & -0.032 to 0.017 & 0.533 \\
\hline$\%$ Eligible completing a Health Check & 0.000 & 0.000 to 0.000 & 0.882 \\
\hline Deprivation score (IMD) & 0.000 & 0.000 to 0.000 & 0.523 \\
\hline Baseline prevalence (2009-2010) & -0.269 & -0.415 to -0.124 & $<0.001^{\mathrm{a}}$ \\
\hline \multicolumn{4}{|l|}{ Chronic kidney disease } \\
\hline $\mathrm{R}^{2}$ & 0.549 & & \\
\hline Adjusted $\mathrm{R}^{2}$ & 0.511 & & \\
\hline Standard error & 0.010 & & \\
\hline Intercept & -0.072 & -0.157 to 0.014 & 0.098 \\
\hline List size & 0.000 & 0.000 to 0.000 & 0.771 \\
\hline Mean age & 0.002 & 0.000 to 0.003 & $0.013^{a}$ \\
\hline$\%$ Male & 0.020 & -0.125 to 0.164 & 0.785 \\
\hline \% Eligible completing a Health Check & 0.000 & -0.001 to 0.001 & 0.974 \\
\hline Deprivation score (IMD) & 0.000 & 0.000 to 0.001 & 0.297 \\
\hline Baseline prevalence (2009-2010) & -0.462 & -0.572 to -0.352 & $<0.001^{\text {a }}$ \\
\hline \multicolumn{4}{|l|}{ Coronary heart disease } \\
\hline $\mathrm{R}^{2}$ & 0.541 & & \\
\hline Adjusted $\mathrm{R}^{2}$ & 0.503 & & \\
\hline Standard error & 0.002 & & \\
\hline Intercept & -0.048 & -0.068 to -0.028 & 0.000 \\
\hline List size & 0.000 & 0.000 to 0.000 & 0.401 \\
\hline Mean age & 0.001 & 0.000 to 0.001 & $0.001^{a}$ \\
\hline$\%$ Male & 0.045 & 0.012 to 0.078 & 0.009 \\
\hline$\%$ Eligible completing a Health Check & 0.000 & 0.000 to 0.000 & 0.188 \\
\hline Deprivation score (IMD) & 0.000 & 0.000 to 0.000 & 0.107 \\
\hline Baseline prevalence (2009-2010) & -0.404 & -0.513 to -0.295 & $<0.001^{\text {a }}$ \\
\hline
\end{tabular}

between the proportion of the population eligible who had a completed Health Check and a change in prevalence for any of the five conditions.

\section{DISCUSSION}

\section{Summary}

Throughout the duration of the study 1142 previously undiagnosed conditions were detected through a total of $16669 \mathrm{NHS}$ Health Checks. Despite this, no significant differences were shown in the change to the prevalence of diabetes, hypertension, $C H D, C K D$, and $A F$ in practices providing NHS Health Checks compared with control practices that were providing usual care over the same period. In addition, regression analysis did not demonstrate any significant association between the proportion of the eligible population of a practice having completed an NHS Health Check and changes in the prevalence of the five conditions studied. The implication of this result is that provision of NHS Health Checks in GP practices may not be different from usual care at increasing the reported prevalence of the five conditions examined despite the apparent detection of disease in $6.85 \%$ of all Health Checks delivered. A further implication was that any cases detected as a result of a Health Check may be equally well detected through usual care. However, it is not possible to determine whether the outcomes for patients who have conditions identified and treated as a result of a Health Check are any different from usual care, or on the wider effectiveness of NHS Health Checks in general.

The strong and consistent negative correlation of lower baseline prevalence of conditions and larger increases in prevalence suggests that practices with a lower initial prevalence find it easier to increase prevalence. This may be because these practices have a larger number of patients with undetected disease than practices with a higher prevalence, the assumption being that the higher the prevalence the more 'complete' the detection of disease within the practice.

The weak but consistently significant correlation between increasing mean age in the practice and increasing prevalence is assumed to be as a result of all five conditions examined being more common with increasing age.

\section{Strengths and limitations}

The main limitation of this study is the small sample size and the resultant underpowering of the study. Although no 
impact of NHS Health Checks on prevalence was shown in this study, an impact cannot be excluded confidently and further larger studies are required. However, this study demonstrates a plausible line of enquiry. It was not possible to expand the sample size because data collection and availability were limited to local practices.

Although the study was controlled, randomisation was not possible because of the manner of the implementation of NHS Health Checks through the national rollout of the programme. Although this does increase the risk of introducing bias and confounding to the results, correction was made for some known confounders in the regression analysis.

The definition of cases for the five conditions examined that were reported to the commissioner of the programme was not explicit. Individual practices reported cases as they diagnosed them according to their usual practice. It is possible, therefore, that some practices underreported cases if their criteria for diagnosis differed from others. There are also some possible differences in the completeness of reporting of cases by practices, but because of limitations in resources there was no ability to verify the data reported by individual practices.

The overall uptake of Health Checks within the study was fairly low at $13.6 \%$ of the eligible population over a 3-year period, an average of $4.53 \%$ per year. This is reasonably similar, however, to the national average uptake of $3.1 \%$ in $2011-2012,{ }^{8}$ increasing to $8.1 \%$ in $2012-2013^{9}$ (mean $5.6 \%$ per year). There is little reason, therefore, to think that the apparent low uptake rate in this study on its own would introduce so much bias as to make the results of this study inapplicable to other parts of the country.

\section{Comparison with existing literature}

This is the first study to examine the impact of NHS Health Checks on the prevalence of disease within practices. Previous researchers have assessed some other aspects of the NHS Health Check scheme such as the impact on cardiovascular risk reduction ${ }^{10,11}$ and the variation in implementation of the programme and uptake among GP practices. ${ }^{12,13}$ There is little available research, however, evaluating the effect of NHS Health Checks on particular conditions, although one study did note that the programme failed to identify one-third of people at high risk of developing diabetes. ${ }^{14}$ A recent Cochrane review showed no effect of general health checks on total or causespecific mortality, although this review did not specifically consider the NHS Health Checks programme. ${ }^{15}$

\section{Implications for research and practice}

This novel study was unable to demonstrate any difference in the changes to the prevalence of diabetes, CHD, hypertension, $C K D$, and $A F$ at a local level in practices offering NHS Health Checks when compared with practices offering usual medical care. However, further research with a larger sample is required to definitively answer this question. It does raise questions, however, in terms of implications for local practice: clinical commissioning groups (CCGs) and public health departments may not be able to rely on NHS Health Checks to reliably case find and increase prevalence of certain conditions over usual care. Studies directly comparing the effect of NHS Health Checks with usual care are lacking and must be the primary focus for further research in this area.

\section{Provenance}

Freely submitted; externally peer reviewed.

\section{Competing interests}

The authors have declared no competing interests.

\section{Acknowledgements}

We thank Dr Kathryn Millard and Nicola Wright in the Warwickshire Public Health department and Chris Buyer in the Arden Commissioning Support Unit for their support with this study.

\section{Discuss this article}

Contribute and read comments about this article: bjgp.org.uk/letters 


\section{REFERENCES}

1. The NHS Health Check Website. About NHS Health Checks. http://www.nhs. uk/Planners/NHSHealthCheck (accessed 9 Jun 2014).

2. Vascular Checks Programme. Putting prevention first. Vascular checks: risk assessment and management. Next steps guidance for primary care trusts. London; Department of Health, 2008.

3. British Heart Foundation. Cardiovascular disease. http://www.bhf.org.uk/ heart-health/conditions/cardiovascular-disease.aspx laccessed 9 Jun 2014).

4. Hippisley-Cox J, Coupland C, Vinogradova Y, et al. Derivation and validation of QRISK, a new cardiovascular disease risk score for the United Kingdom: prospective open cohort study. BMJ 2007; 335: 136.

5. The NHS Health Checks website. NHS Health Checks Research. http://www. nhshealthcheck.nhs.uk/default.aspx?ilD=22\&alD=115\&st=research laccessed 9 Jun 2014).

6. The Health and Social Care Information Centre. Quality and Outcomes Framework. http://qof.hscic.gov.uk/index.asp laccessed 9 Jun 2014).

7. Faul F, Erdfelder E, Buchner A, Lang A-G. Statistical power analyses using G*Power 3.1: tests for correlation and regression analyses. Behav Res Meth 2009; 41: 1149-1160.

8. NHS England. NHS Health Checks Data 2011/12. http://transparency. dh.gov.uk/files/2012/07/IPMR_1-for-web-Health-Checks-Q4-2011-12- revised-14.11.2012.xls laccessed 9 Jun 2014)

9. NHS England. NHS Health Checks Data 2012/13. http://www.england.nhs. uk/statistics/wp-content/uploads/sites/2/2013/04/Health-Checks-webfile-Q4-2012-13.xls (accessed 9 Jun 2014).

10. Cochrane T, Davey R, Iqbal Z, et al. NHS health checks through general practice: randomised trial of population cardiovascular risk reduction. BMC Public Health 2012; 12: 944.

11. Artac M, Dalton AR, Majeed A, et al. Effectiveness of a national cardiovascular risk assessment program (NHS Health Check): results after one year. Prev Med 2013; 57(2): 129134.

12. Graley C, May K, McCoy D. Postcode lotteries in public health - the NHS Health Checks Programme in North West London. BMC Public Health 2011; 11: 738 .

13. Dalton A, Bottle A, Okoro C, et al. Uptake of the NHS Health Checks programme in a deprived, culturally diverse setting: cross-sectional study. J Public Health 2011; 33(3): 422-429.

14. Smith S, Waterall J, Burden AC. An evaluation of the performance of the NHS Health Check programme in identifying people at high risk of developing type 2 diabetes. BMJ Open 2013; 3: e002219. doi:10.1136/bmjopen-2012-002219.

15. Krogsbøll L, Jørgensen K, Larsen C, Gøtzsche P. General Health Checks in adults for reducing morbidity and mortality from disease: Cochrane systematic review and meta-analysis. BMJ 2012; 345: e7191. 